\title{
Self-dissimilarity, irreversibility and robustness in mode-locked fiber oscillators
}

\author{
Ghaith Makey, ${ }^{1}$ Tesfay Teamir, ${ }^{1}$ Serim Ilday ${ }^{2}$, and F. Ömer Ilday ${ }^{1,2,3 *}$ \\ ${ }^{1}$ Department of Physics, Bilkent University, 0680 Ankara, Turkey \\ ${ }^{2}$ UNAM-National Nanotechnology Research Center and Institute of Materials Science and Nanotechnology, Bilkent University, 0680 \\ Ankara, Turkey \\ ${ }^{3}$ Department of Electrical and Electronics Engineering, Bilkent University, 0680 Ankara, Turkey \\ Corresponding author: ilday@bilkent.edu.tr
}

\begin{abstract}
We introduce self-dissimilarity as measure of phase space complexity and predictor of robustness against perturbations. As nonlinearity increases, phase space becomes a random fractal, just before critical transitions. Measurements confirm powerlaw dependence over 7 decades. (C) 2018 The Author(s)
\end{abstract}

OCIS codes: (060.3510) Lasers, fiber; (140.4050) Mode-locked lasers

\section{Introduction}

Passively mode-locking of a laser is a self-organized steady state born out of fluctuations, reaching a farfrom-equilibrium steady state. At low nonlinearity, mode-locking constitutes a stable state with low noise, i.e., external fluctuations are strongly damped. At high nonlinearities, things becomes more complicated. All sorts of instabilities and critical transitions have been observed over the past decades. This is especially true for fiber lasers, which, together with their huge gains and large nonlinearities due to confinement of light in the cores of meters-long pulses. However, even at low powers, corresponding to relatively weak nonlinearities, mode-locking can become destabilized by various perturbations, such as thermal fluctuations or mechanical vibrations. It is curious that there has not been much discussion about the origins of robustness, despite the fact that every practitioner in the field knows that certain mode-locking regimes, e.g., similariton and all-normal dispersion (supporting dissipative solitons) appear to be more robust than, e.g., soliton and stretched-pulse regimes under comparable conditions. Why is this the case? Can it be explained? In addition its enormous practical relevance, any potential answer may hold implications for broad classes of selforganized states in diverse fields of science.

Here, we introduce self-dissimilarity of the phase space of mode-locking as a measure of its underlying complexity, which we characterize experimentally for the four main regimes of mode-locking. The reasoning is straightforward: When the laser is perturbed, this changes in the optical parameters of the cavity. If the new setting supports a mode-locking state that is virtually identical to the original state, the fact that a perturbation has occurred is inconsequential. Then, if the laser's phase space is occupied by the same state, the probability of a perturbation not changing the original state is higher. In contrast, if the phase space is occupied by a very large number of distinct states, each occupying tiny areas, then almost every perturbation causes the laser to operate differently, which we perceive as lack of robustness. Borrowing the complexity measure of selfdissimilarity from complex systems, we apply it here to quantify how varied the phase. We also distinguish between two scenarios, if the mode-locking state is changed in response to a perturbation because many perturbations, such as fluctuations in pump power, will average to zero over some time scale. If the transition from the original state to the new state is reversible, then upon the perturbed value, say, pump power, returning to its original value, the laser will revert to its original mode-locking state. For many situations of practical importance, the fact that a transition has occurred may not matter if the perturbed value average zero on a sufficiently fast time scale. Therefore, robustness of a mode-locking state is expected to be higher if the selfdissimilarity and irreversibility of the mode-locking transitions are low.

We use a nonlinear polarization evolution (NPE) based Yb-doped fiber laser to demonstrate these ideas. The laser could be modified to operate in each of the four well known regimes and the waveplates are used to explore the phase space (changing one of the waveplates is similar to a mechanical perturbation on the fiber). Optical autocorrelation (AC) and optical spectrum (OS) traces are used to judge whether the laser is mode locked or not and if the mode-locking state changes. These data recorded digitally as two of the waveplates were rotated over a range, as shown Fig. 1. If the optical spectrum and autocorrelation traces differ by more than a threshold amount, then the mode-locking state is taken to be different. The precise value of this threshold does not alter any of the conclusions present here. Having made the characterization for the different mode-locking regimes, the differences are evident even visually. Similariton and dissipative soliton regimes are characterized by larger areas occupied by a smaller set of mode-locking states. We quantify this by calculating a self-dissimilarity coefficient $(\eta)$ defined as $\eta=\sum_{k=0}^{n}\left(\frac{x^{k}}{x^{0}}\right)-1$, where $x^{0}$ and $x^{k}$ are the areas of the largest mode-locking state, and the $k^{\text {th }}$ one within phase space respectively. Self-dissimilarity is 
lowest $(0.1)$ for the similariton regime and 0.8 for the dissipative soliton (all-normal dispersion) regime. This is presumably due to the presence of a strong similariton attractor that does not depend on the initial conditions [5] and relatively stronger dissipation [6]. It is 2.7 for dispersion-managed and even higher (6.7) for the soliton regimes. Similarly, irreversibility is highest for the latter two and lowest for the dissipative soliton regime.

Furthermore, we find that regardless of the mode-locking state, all regimes we have tested universally develop a complex phase space (highly self-dissimilar) as nonlinearity is increased - albeit at quite power levels. With increasing nonlinearity, the phase space develops into a random fractal, which we have experimentally verified to exhibit a power-law dependence over 7 decades (Fig. 2). This finding explains the fundamental mechanism through which a well-known fact is experienced; namely, that all mode-locked lasers become "finicky" as nonlinearity is increased.

In conclusion, we propose self-dissimilarity as a measure of complexity of mode-locking states for laser oscillators and together with irreversibility of transitions, it successfully predicts and explains the origins of higher robustness. We also find that all mode-locking regimes develop critical transitions through evolution of their phase space into a random fractal as laser power, therefore, nonlinearity is increased.

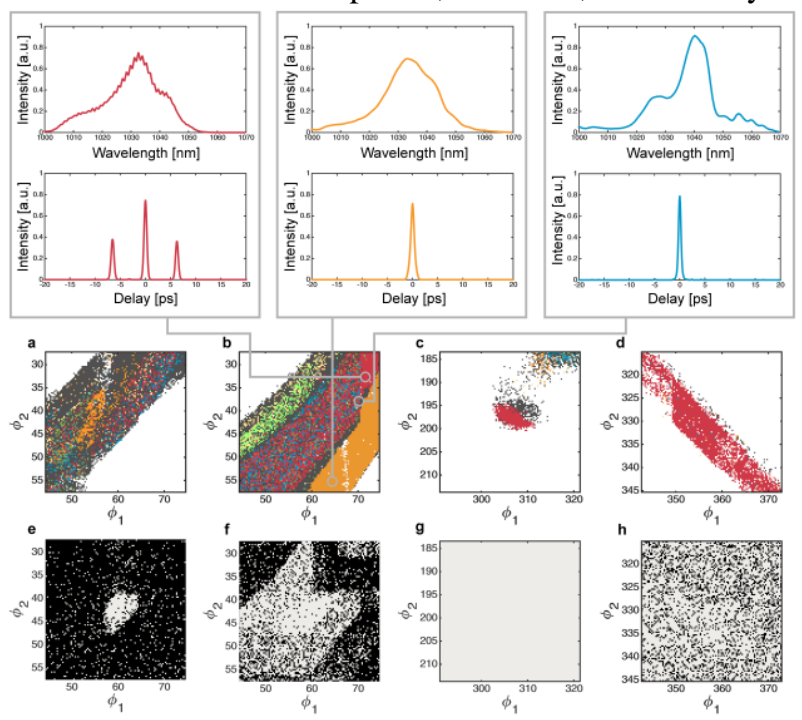

Fig. 1: Phase space map obtained by scanning $30^{\circ}$ by each waveplate with a resolution of $0.3^{0}:$ (a-d) Phase space formed by cross-correlation of optical spectrum and autocorrelation signal of lasing states for soliton, dispersion-managed soliton, dissipative soliton (all-normal dispersion) and similariton regimes, respectively. The 5 most common states are each assigned a separate color and the remaining ones are shown as gray points; (e-h) reversibility maps of each state for soliton, dispersion-managed soliton, dissipative soliton and similariton regimes, respectively.
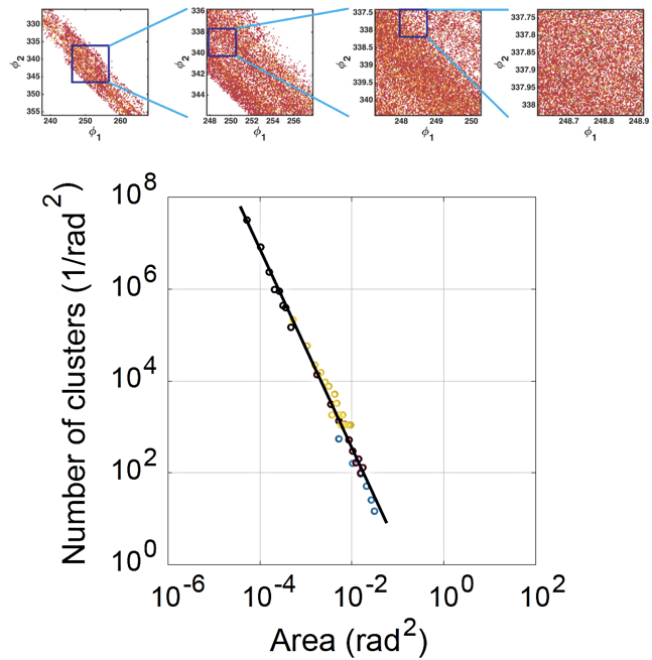

Fig. 2: By increasing nonlinearity, the phase space develops into a random fractal, which we have experimentally verified to exhibit a power-law dependence over 7 decades.

\section{References}

[1] C. Song, S. Havlin and H. Makse, "Self-similarity of complex networks," Nature 433, 392 (2005).

[2] John M. Dudley, Christophe Finot, David J. Richardson and Guy Millot, "Self-similarity in ultrafast nonlinear optics," Nature Phys. 3,597 (2007) 